\section{G161(P) RETROSPECTIVE ANALYSIS OF THE ESTABLISHMENT OF NON-INVASIVE VENTILATION IN CHILDREN}

doi:10.1136/archdischild-2013-304107.173

S Hodgson, A Morley, NA Gibson, PL Davies. Respiratory Medicine, Royal Hospital for Sick Children, Glasgow, UK

Aims Non-invasive ventilation (NIV) is now an established treatment for children with sleep disordered breathing. We report our experience of starting children on NIV over 12 years and aim to identify areas for future improvement.

Methods We retrospectively analysed the data of all children we attempted to established on NIV in our centre between July 2000 and July 2012. We noted the clinical indication, whether NIV was successful and, if available, any sleep study results obtained.

Results NIV was attempted in 122 patients. 46 (37.7\%) had obstructive sleep apnoea (21 related to obesity); 26 (22.1\%) neuromuscular disease (2 additional children with SMA1 had a brief trial of NIV); 16 (13.1\%) Down's syndrome; 11 (9\%) craniofacial or skeletal disorders; 7 (6\%) central hypoventilation; 5 (4\%) mucopolysaccharidoses; 8 (7\%) had other disorders.

$66 / 122$ had pre and post-establishment oximetry performed with $92.4 \%$ showing improvement in desaturation index (DSI) (mean DSI was 20.7 pre-establishment, and 2.5 post-establishment, $\mathrm{p}<0.01$ )

111 children (91\%) were successfully discharged from hospital on NIV. Of 11 children who failed to establish 7 had Down's syndrome, 2 others had SMA1 and the families chose not to proceed. Median patient age was 10.61 years (range 0.71-18.17), whilst median age of those that failed to establish was 5.95 years.

Of 111 children discharged on NIV, 12 (10.8\%) stopped due to poor acceptance of treatment (4 of these had Down's syndrome), 54 $(48.6 \%)$ continue on NIV within paediatrics, 20 (18\% ) have transferred to adult services, 9 (8.1\%) have stopped due to an improvement in their medical condition over time, 7 (6.31\%) stopped as no clinical improvement was achieved with therapy, 6 (5.41\%) have died and $3(2.7 \%)$ have been lost to follow up.

Conclusion Overall long-term success rates of NIV were high, with the majority of children continuing on ventilation under paediatric or adult services or stopping due to clinical reasons. The main reason for failure of establishment or maintenance was nonacceptance. This was significantly greater in children with Down's syndrome and new strategies must be found to improve acceptance with these patients.

\section{G162(P) WHAT PREDICTS DURATION OF HOSPITAL STAY FOR BRONCHIOLITIS?}

doi:10.1136/archdischild-2013-304107.174

R Watt, G Jackson, S Turner. Child Health, University of Aberdeen, Aberdeen, UK

Background Bronchiolitis is a common lower respiratory tract infection of infancy. In theUK, $3 \%$ of all infants are hospitalised with bronchiolitis and this places a considerable burden on the NHS every winter. An admission costs $£ 600 /$ day in our institution. Identification of factors associated with the duration of hospitalisation with bronchiolitis may be of interest to clinicians and healthcare commissioners and this was the aim of the present study.

Methods Data were collected from infants aged up to 6 months and admitted to hospital with a diagnosis of bronchiolitis during the winters 2003/4-2010/11 inclusive. The outcome was duration of stay (hours). Predictive variables included weight, age and observations on admission, gender, socioeconomic status, distance from home to hospital, number of infants admitted that winter and winter of admission. A clinical pathway was introduced after winter $2004 / 5^{1}$.

Results Data were available in 382 infants, 322 were RSV positive. The median (IOR) duration of stay was 66 hours $(38,99)$. Initial univariate analyses indicated that the following were positively associated with duration of stay: younger age (Rho-0.31), lower weight (Rho-0.31), increased heart (Rho0.17) and respiratory rate (Rho0.18), detection of RSV (mean duration of stay 84 for RSV positive vs 48 hours for RSV negative) and smaller number of infants admitted that winter (Rho-0.17). The duration of stay also varied by year and was reduced after introduction of the clinical pathway ${ }^{1}$. In the multivariate analysis $\left(\mathrm{R}^{2}=0.29, \mathrm{n}=347\right)$, the following remained significantly associated with log transformed duration of stay: respiratory rate $(p=0.003)$, infant weight $(p<0.001)$, RSV detected $(p<0.001)$ and winter of admission $(p<0.001)$.

Conclusions The duration of stay for bronchiolitis, measured over eight successive seasons, appeared to be partly driven by host and pathogen factors. There are differences between years, e.g. a "busy" season, introduction of a clinical pathway which may lead to a reduction in duration of stay. However, most of the variation in the duration of stay within this population was explained by variables not measured and may include random factors.

\section{REFERENCE}

1. Walker C, Danby S, Turner SW. Impact of a bronchiolitis clinical care pathway on hospital stay. Eur J Pediatr 2012; 171:827-832.

\section{G163(P) RECURRENT LOWER RESPIRATORY TRACT INFECTION (LRTI) IN CHILDREN WITH REPAIRED TRACHEOESOPHAGEAL FISTULA (TOF)}

doi:10.1136/archdischild-2013-304107.175

'B Nasrallah, 2B Jaffray, 'S Moss. 'Paediatrics Respiratory Department, Great North Children's Hospital, Newcastle Upon Tyne, UK; '2Paediatrics Surgical Department, Great North Children's Hospital, Newcastle Upon Tyne, UK

Aim To identify if patients with repaired TOF have increased risk of recurrent LRTI

Methods We reviewed the medical records of all children who had repaired TOF over the last 20 years (1990-2011) in our centre. We identified the children who presented with recurrent LRTI. We reviewed their medical records and radiological tests and findings.

Results 82 children had repaired TOF over 20 years period. Age range from 1 - 20 years old post repair period. 18 children (22\%) had recurrent LRTI and needed regular courses of antibiotics. 10 out of 18 children had high resolution CT scan (HRCT) of the chest and 3 of them (4\%) had confirmed bronchectasis.

Conclusion While tracheomalacia is a well recognised complication of TOF repair, there is paucity of data regarding the increased risk of LRTI. One recent study demonstrated increased cough with sputum production and chronic bronchitis in repaired TOF. (Acta Paediatr. 2011 Sep; 100(9):1222-5).

In our study we found increased risk of recurrent LRTI in children with repaired TOF (one fifth of the patients). The incidence of bronchectasis was $4 \%$. These findings highlights the importance of awareness of the long-term respiratory complications in children with repaired TOF.

\section{G164(P) LOCAL DEMOGRAPHIC OF PAEDIATRIC ASTHMA VISITORS AT ACCIDENT AND EMERGENCY AT A DISTRICT GENERAL HOSPITAL}

doi:10.1136/archdischild-2013-304107.176

'D Kular, ${ }^{2} \mathrm{R}$ Chodhari. 'Department of Paediatrics and Child Health, University College London, London, UK; ${ }^{2}$ Department of Paediatrics and Child Health, The Royal Free London Foundation NHS Trust, London, UK

Aims According to Asthma UK: an estimated 75\% of admissions are avoidable. Although, national data is available there is paucity of local data. We have aimed to produce a local demographic of asthma 